\title{
"Se busca"
}

\section{Métodos de investigación para una aproximación feminista a mujeres detenidas por la policia}

\author{
"Wanted" \\ Research methods for a feminist approach to women in police \\ detention
}

\author{
Estibaliz de Miguel Calvo \\ Universidad del País Vasco / EHU \\ estibaliz.demiguel@ehu.eus
}

Recibido: 12.062019

Aceptado: 20.02.2021

\section{RESUMEN}

Este artículo explora cómo es posible poner en práctica métodos de investigación para analizar la experiencia de las mujeres detenidas en calabozos desde una perspectiva feminista. Presentamos el proceso metodológico desarrollado con las mujeres que pasaron por una experiencia de detención policial, prestando especial atención a las cuestiones éticas. Para ello, se realiza un análisis de la literatura sobre este objeto de investigación, se describe el contexto de la investigación, la metodología desarrollada y las decisiones tomadas sobre las técnicas de investigación más apropiadas al contexto concreto y las especificidades de las mujeres detenidas por la policía. Se ilustra la utilidad de la reunión de cierre con un doble propósito: sopesar el impacto de la investigación para las participantes y devolver los resultados. Pero el grupo de cierre posibilitó cumplir con otros objetivos no contemplados inicialmente, tales como el contraste grupal de resultados, la rendición de cuentas acerca de la marcha de la investigación y la generación de dinámicas de empatía y validación mutua En definitiva, el grupo de cierre fue mucho más allá de las expectativas iniciales, sirviendo de aprendizaje para las investigadoras acerca del enorme potencial de las técnicas grupales para el estudio de experiencias de mujeres.Más allá de lo inicialmente previsto, el grupo de cierre permitió establecer otras formas de relación con las mujeres participantes reduciendo la distancia investigadoras/participantes y facilitando formas más horizontales de generar conocimiento. 


\title{
PALABRAS CLAVE
}

Metodología feminista, métodos cualitativos, ética de la investigación, sistema penal, detención policial, mujeres criminalizadas. Criminología.

\begin{abstract}
This article explores how research methods can be used to analyse the experience of women in police custody from a feminist approach. Here, there are presented the methodological process developed with women who went through an experience of police detention, paying particular attention to ethical issues, through a review of the literature on this object of research, a description of the research context, the methodology developed and the decisions made about the most appropriate research techniques for the specific context and the specificities of women in police detention. The usefulness of the closing meeting is illustrated with a double purpose: to weigh the impact of the research for the participants and to return the results, But the closing group made it possible to fulfil other objectives not initially envisaged, such as group comparison of results, accountability on the progress of the research, as well as the generation of dynamics of empathy and mutual validation. In short, the closing group went far beyond the initial expectations, serving as a learning experience for the researchers about the enormous potential of group techniques for the study of women's experiences. Beyond what was initially envisaged, the focus group allowed for the establishment of other forms of relationships with the women participants, reducing the researcher/participant distance and facilitating more horizontal ways of generating knowledge.
\end{abstract}

\section{KEY WORDS}

Feminist methodology, qualitative methods, research ethics, penal system, police detention, criminalized women, criminology.

\section{INTRODUCCIÓN}

"Se buscan mujeres que hayan sido detenidas por la policía en la Comunidad Autónoma para participar en una investigación". Así comenzaba el llamado por mail y redes sociales para contactar con mujeres que hubiesen pasado por alguna de las comisarías vascas. Esta fue una de las tres estrategias de contactación implementadas, junto con el reclutamiento a través de entidades sociales o abogados y abogadas, y la técnica de bola de nieve, con el objetivo de conocer la experiencia de detención policial de mujeres en primera persona. A este llamado respondieron 9 mujeres en el plazo de 6 meses de realización del trabajo de cam- 
po, un $14,5 \%$ de todas las partes (junto con abogados y abogadas, movimientos sociales y policías) que entraron en juego para dibujar el retrato de la experiencia de detención policial a mujeres.

Además del enorme reto que suponía contar con la participación de las mujeres detenidas, varias cuestiones estuvieron presentes desde el inicio del proceso. Una pregunta general ¿cómo es posible poner en práctica métodos de investigación con enfoque feminista para analizar la experiencia o los discursos de las mujeres detenidas en calabozos? Y dos preguntas concretas de carácter ético: ¿qué impacto personal y emocional tiene relatar la experiencia de detención policial en primera persona? ¿qué pasos dar a lo largo del proceso para acompañar el posible daño de revivir esa experiencia? Este es, precisamente, el objeto del presente artículo: describir las dudas que se plantearon y las decisiones tomadas con respecto a la participación de mujeres que han sido detenidas con un doble propósito. Por una parte, dilucidar los métodos que mejor se ajustaran a ese marco feminista y a los objetivos de la investigación, teniendo en cuenta que no existe un método propiamente feminista (Harding 1987), pero sí diversas técnicas y procedimientos que permiten dar más voz a las protagonistas, al tiempo que se aminoran las relaciones de poder intrínsecas en toda investigación. Y por otra, el presente texto tiene por objeto describir las decisiones tomadas en la búsqueda de aminorar en lo posible el daño que pudiese generar a las protagonistas el hecho de participar en la investigación, proporcionando para ello un entorno de cuidado en la investigación, más allá de los límites marcados por los códigos éticos de las ciencias sociales y la criminología (Viedma Rojas et al. 2019). En este punto residía el "se busca". Para abordar esta búsqueda redoblamos esfuerzos en tiempo y recursos humanos ${ }^{1}$.

De esta manera, se planteaba que la investigación fuera feminista no sólo desde un punto de vista teórico, sino también metodológico, es decir, por las premisas de partida y las consideraciones que se incluyen en el proceso, que tienen en cuenta el eje de género y otros ejes de desigualdad que intersectan en las vidas de las mujeres en el sistema de justicia penal. Atender a las cuestiones metodológicas supone prestar atención no solo a resultados, sino también a las implicaciones del proceso. Así, se abre la posibilidad a reflexionar sobre nuestra posición como investigadoras, sobre el impacto de la investigación en el colectivo estudiado y de pensar cómo se generan los resultados a través de las decisiones tomadas por el equipo investigador, al tiempo que se atiende a interacción con individuos, situados en un determinado contexto social e institucional. Las reflexiones que aquí se presentan sobre el abordaje feminista de la investigación de detención policial a mujeres se encuadra en una investigación más amplia. A saber, la investigación sobre la experiencia de detención policial a mujeres en la Comunidad Autónoma de Euskadi (CAE), que combina una metodología cuan-

${ }^{1}$ El equipo de trabajo formado por Lohitzune Zuloaga Lojo (coordinadora) (UPNA), Estibaliz de Miguel Calvo (UPV/EHU) y Miren Ortubay Fuentes (UPV/EHU), contó con la asesoría de Itziar Gandarias Goikoetxea (UD) y el apoyo de Natacha Guala (Universidad de El Litoral, Argentina). 
titativa de estudio de las estadísticas policiales, con una metodología cualitativa que diera cuenta de la voz de diferentes agentes implicados en la experiencia de detención policial de mujeres, no solo a quienes habían estado detenidas en comisaría, sino también agentes de policía, profesionales de la abogacía del turno penal y entidades sociales que de trabajen con personas susceptibles de ser detenidas por la policía.

En este artículo trata exclusivamente los retos, reflexiones y decisiones tomadas en relación al abordaje metodológico de la experiencia de detención en el caso de aquellas mujeres que lo habían vivido en primera persona. Aunque el número de mujeres que pasaron por una experiencia de detención policial fue claramente menor respecto a otros agentes $(9$ mujeres entrevistadas frente al total de 62 entrevistas), se dedicó tiempo y esfuerzo a la reflexión acerca de los métodos y el procedimiento de investigación más adecuado con las protagonistas por dos razones principalmente. Primero, debido a que la experiencia de detención policial tiene consecuencias subjetivas y sociales para aquellas mujeres que pasan por comisaría, que hace de ellas "sujetos de alto riesgo o vulnerables" (Schlosser 2008). Y segundo, el proceso rememorar una experiencia que puede resultar estresante o dolorosa (Grau 2015), algo que se quería aminorar en la medida de lo posible.

Escasa atención se ha dedicado en la literatura científica a la experiencia cotidiana de la detención policial, más allá de flagrantes violaciones de derechos humanos, menos aún en lo que a las mujeres se refiere. Los trabajos publicados en nuestro contexto acerca de la detención policial se centran sobre todo en casos de tortura y vulneraciones de derechos humanos descritos por entidades de lucha por los derechos humanos u organismos internacionales (Comité para la Prevención de la Tortura 2017; Etxeberria, Beristain y Pego 2016; RIS 2019; San Martín et al. 1991), vulneraciones que se producen más a menudo durante el periodo de detención incomunicada (Pego Otero 2017) que durante el tiempo ordinario de detención. La actividad de la policía ha sido también criticada por su sesgo racista en lo que a las detenciones selectivas a afrodescendientes se refiere (Orgaz Alonso, 2018, Asamblea General de la ONU 2018, Sainz de la Maza Quintanal, 2015; BVODDHH, 2013).

Las disciplinas que han estudiado la configuración y práctica del sistema penal tienden a adolecer de una mirada androcéntrica, salvo la atención otorgada por las disciplinas dedicadas a estas cuestiones con una perspectiva crítica de género. La criminología feminista nace en el contexto anglosajón durante los años 70 incorporando la perspectiva de género para dar visibilidad a las mujeres en los estudios sobre delincuencia (Smart 1977). El interés de la nueva corriente se centró en estudiar la condición de desigualdad de las mujeres, bien como autoras de delitos, bien como víctimas de ellos, poniendo de manifiesto que las trayectorias y circunstancias que llevan a las mujeres a cometer delitos son cualitativamente diferentes a las de los hombres (Heimer y Kruttschnitt 2005; Barberet 2014), desenmarañando cómo se articulan las estructuras básicas de opresión (patriarcado y capitalismo) en nuestras sociedades contemporáneas. Con estas premisas, la criminología feminista ha hecho visibles a las mujeres víctimas y a 
las delincuentes, al tiempo que ha estudiado el sexismo institucionalizado en las prácticas, asunciones teóricas y políticas llevadas a cabo por los diversos agentes de control social. Este cambio de perspectiva ha conducido a la disciplina a prestar atención a cuestiones que afectan específicamente a las mujeres, como son el miedo a la violencia, la sexualidad, o la ideología que asigna un determinado papel a la mujer en la sociedad; interpretándolos como «mecanismos de control peculiares, dirigidos a las mujeres» (Larrauri 1992).

En el contexto español, la delimitación de la disciplina criminológica ha tenido una deriva particular debido a las diferentes correlaciones de fuerzas académicas, donde ha predominado un quehacer de tintes marcadamente jurídicos, más que sociológicos (Miranda y Sánchez 2018). Han sido investigadoras en los campos de la sociología jurídica o la sociología del delito y las prisiones quienes han desarrollado trabajos con mayor enraizamiento en las ciencias sociales y con una perspectiva crítica feminista del sistema penal, donde han predominado las metodologías cualitativas. Estos trabajos se han llevado a cabo especialmente a partir del advenimiento del siglo XXI (Almeda 2017), convocadas por redes de trabajo como GEISPE-Red Internacional de Género y Sistema Penal. Pero se han centrado predominantemente en las sentencias firmes de mujeres condenadas, prestando especial atención a ese porcentaje menor del 10\% que suponen las mujeres entre población encarcelada a nivel mundial (Walmsley 2015), más que al estudio empírico de otros eslabones del sistema penal. A pesar de ello, resulta llamativo que, siendo las cárceles instituciones totales (Goffman 2001) que presentan no pocos retos para la investigación social, los trabajos de nuestro contexto no hayan pasado de algunas referencias puntuales a los retos metodológicos planteados (Sádaba Rodríguez 2014; Viedma Rojas Del Val Cid y Callejo Gallego, 2019, en centros penitenciarios y Orgaz Alonso, 2018 en Centros de Internamiento de Extranjeros), y muy puntualmente se trata de reflexiones metodológicas feministas (Ballesteros-Pena, 2019; De Miguel Calvo 2016).

Tras descartar otras técnicas que requerían más tiempo, más recursos y mayor exigencia tanto para las investigadoras como las participantes, optamos por la entrevista en profundidad semiestructurada con un formato aplicado a las circunstancias específicas de las experiencias de las mujeres que han pasado por comisaría. Para sopesar el impacto subjetivo y emocional que el relato de la experiencia de detención policial podía tener, diseñamos un grupo de cierre al final del proceso, en que se convocase a todas las participantes en las entrevistas para valorar lo que había supuesto narrar su experiencia en comisaría. Al proceso de elección de métodos de abordaje de la experiencia en primera persona de la detención policial de mujeres, y más concretamente, al grupo de cierre y sus implicaciones, nos dedicaremos más extensamente al final del presente artículo. Antes, describiremos el marco feminista de investigación y sus implicaciones éticas para el tema de estudio y las participantes protagonistas de una detención policial. También nos dedicaremos a los rasgos más relevantes de la investigación sobre la experiencia de detención policial a mujeres en la CAE, y dentro de ella, las limitaciones a las que nos veíamos sometidas tanto por parte de las 
condiciones marcadas por la institución que patrocinaba el proyecto, como por los rasgos de los perfiles de las investigadoras.

\section{ENFOQUE Y ÉTICA FEMINISTA PARA CONOCER LA EXPERIENCIA MUJERES EN DETENCIÓN POLICIAL}

La metodología feminista es un conjunto de enfoques que abordan el problema de producir conocimiento acerca de las relaciones de género. Muchos de sus postulados convergen con las propuestas que se alejan de las asunciones del conocimiento positivista. Lo que caracteriza a las corrientes feministas es que han sido críticas con la forma de producir conocimiento científico de la vida social que decía ser neutral políticamente, o neutro al género, mientras que en la práctica promocionaba, reproducía o ignoraba la apropiación masculina de la ciencia y la razón (Ramaznoglu y Holland 2002). Para Bárbara Biglia (2015), esta crítica al sesgo sexista que se esconde bajo la neutralidad y la objetividad que defiende la ciencia positivista, se traduce en una apuesta por la asunción de responsabilidad por parte de las investigadoras en la producción de conocimiento y el reconocimiento, por tanto, de que producir conocimiento es siempre un acto político.

De esta manera, como afirman Ramaznoglu y Holland (2002), lo que caracteriza a la metodología feminista no es principalmente que está hecha por mujeres (también puede ser producida por hombres) o que usa unas determinadas técnicas (aunque tiende a usar las cualitativas para captar las experiencias), tampoco porque haya una posición ontológica o epistemológica propia (ya que puede variar desde el realismo hasta el relativismo). Lo que distingue a la metodología feminista es que es coherente con los postulados feministas, la posición política y ética feminista basada en las experiencias de las mujeres, y que rechaza las "verdades" patriarcales que defienden que las mujeres son naturalmente inferiores a los hombres, oponiéndose a los razonamientos y los métodos científicos que no tienen en cuenta las desigualdades de género.

En esta línea, Margaret Malloch (2000) formula los pilares básicos de enfoque crítico feminista. Primero, el tema de estudio ha de suponer algún tipo de reconocimiento del género y sus relaciones de poder, poniendo el acento principalmente en la posición de las mujeres. Segundo, los métodos deben posibilitar a quien investiga lograr una visión de la experiencia individual de la persona participante, pero al mismo tiempo permite colectivizar las experiencias individuales en la búsqueda de un cambio social. Tercero, la relación entre el o la investigadora y la investigada debe ser lo menos jerárquica posible. Y cuarto, la investigadora feminista se localiza claramente dentro de las actividades de la investigación, por tanto, no permanece ausente como si de una voz en off se tratara.

Los planteamientos feministas de investigación han tenido un proceso de maduración que ha permitido revisar las premisas iniciales y matizar algunos de sus postulados, enriqueciendo el planteamiento. En esta línea, Hammersley 
(1992) revisa los elementos clásicos de la metodología feminista: el género como categoría de desigualdad, la atención a la experiencia, el rechazo a las jerarquías, la centralidad de las mujeres y el horizonte de la emancipación. Para empezar, es necesario tener en cuenta que además del género hay que introducir otros ejes de desigualdad en el análisis, como la clase social y la raza, lo cual nos lleva a la justificación del análisis interseccional que proponemos aquí. En relación a la experiencia frente al método, el autor advierte del peligro de reificación de la misma y recuerda que la experiencia también es construida. El rechazo de las jerarquías es también problemático, ya que se asume que el poder es unidireccional investigadora-participante, dejando de lado otras dinámicas de poder. Además, el estudio no siempre se focaliza en mujeres, ya que cabe estudiar la construcción de las masculinidades o los contextos institucionales de gestión de diversos aspectos que afectan a las cuestiones de género. Finalmente, el objetivo de emancipación quizás sea el más problemático ya que tiende a ser demasiado general y muy complejo de conceptualizar, si tenemos en cuenta la maraña de relaciones de poder en que las personas se encuentran en la compleja combinación de categorías de género, raza, clase social, edad, entre otras.

La consideración de las personas más allá de meros objetos de investigación sino de sujetos con capacidad reflexiva y activa como parte de la centralidad de la dignidad humana en el quehacer científico, implica considerar aspectos relativos a las cuestiones éticas implicadas, especialmente teniendo en cuenta que en nuestro caso estamos tratando con lo que se consideran "temas sensibles de investigación" (Dickson-Swift et al., 2008)囚. En las ciencias sociales, las cuestiones éticas se empezaron a desarrollar en los años 70 y a lo largo de los 80 , con la proliferación de comités éticos y diferentes códigos y declaraciones que coinciden en identificar tres principios fundamentales: el consentimiento informado de las personas participantes, la confidencialidad de la información recibida y el anonimato de los sujetos estudiados (Meo 2010).

Abad (2016) apunta que existen grandes dificultades para poner en práctica estos tres principios en el contexto de la investigación cualitativa, concluyendo que es necesaria una ética situada o micro-ética, basada en procesos y en la implicación activa de quienes participan. Así, el consentimiento informado más allá de un momento puntual de explicación de la investigación y posterior aceptación de participar, se concibe en movimiento, esto es, construido, negociado y reafirmado continuamente entre las personas participantes. Frente a la idea de la "fabricación de datos", reconocer que el significado es dialógico, lo que quiere decir que se produce en la interacción entre, al menos, dos partes (investigadora y participante) en un momento y lugar concreto. Finalmente, frente a la concepción de la figura de la participante como objeto de investigación, el reconocimiento de la otra parte como sujeto interlocutor de pleno derecho.

Además de estas tres consideraciones básicas, resulta primordial considerar el daño potencial de la investigación en las personas participantes (Lipson 2003). En nuestro caso, y como venimos señalando, nos preocupaba de manera especial el impacto que pudiera tener en las mujeres que han pasado por una experiencia de detención policial hacer memoria y revivir ese acontecimiento, ya que puede 
suponer un viaje emocional duro que quienes investigamos deseamos acompañar con responsabilidad. Smith y Pittis (2005) definen una serie de líneas para la investigación de grupos en situación de vulnerabilidad que han servido de orientación para el trabajo de investigación que aquí se presenta: evitar el daño, beneficencia o hacer bien, respeto por las personas más allá del consentimiento informado, y la noción de representación, es decir, de qué manera una determinada comunidad es representada. Además, es importante tener en cuenta el principio de reciprocidad, que implica una responsabilidad de las personas con respecto a las investigadas.

En el caso de la metodología feminista, la dimensión ética tiene una presencia explícita y es una de sus señas de identidad sobre todo si tenemos en cuenta que, como afirman Ramaznoglu y Holland: "lo que distingue a las investigadoras feministas es que comparten un compromiso ético y político que les hace responsables frente a una comunidad de mujeres con intereses morales y políticos en común" (2002:16). Ackerly y True definen el compromiso ético feminista como "el compromiso de investigar cómo investigamos" (2010:22). Esto requiere prestar atención a las relaciones de poder y sus efectos, en lo que se refiere a aspectos como las formas de poder existentes en los planteamientos epistemológicos (positivistas-masculinos) acerca de producción de análisis de la realidad; los límites disciplinarios existentes en las categorías sociales como el género, la clase y la raza; las diversas relaciones que se generan en el proceso de investigación (investigadora-participante, en el equipo investigador, el contexto de investigación donde se producen las interacciones, etc.), y la posición de las investigadoras, una posición que quiere ser reflexiva sobre sí mismas y su posición sociopolítica en el proceso de investigación.

Atender a las relaciones de poder implica entender la investigación como un proceso dialógico donde investigadora y participantes se influyen mutuamente y adquieren diferentes posiciones de poder y privilegio a lo largo de la investigación. De esta manera, situación investigada es estructurada tanto por la investigadora como por las participantes (England 1994).

Por otra parte, en el encuentro entre investigadoras y participantes se reconocía el valor de las emociones como recurso intelectual (Jewkes 2011), por muy paradójico que esto pueda parecer desde parámetros de raciocinio dicotómicos. Las emociones son algo presente en el quehacer investigador, ya sea por parte de las investigadoras quienes experimentan excitación, ansiedad, curiosidad o placer durante el desempeño de su labor; como por parte de las personas informantes, quienes pueden recelar o desconfiar, pero también sentir alivio y reparación al ser escuchadas, incertidumbre por conocer los resultados de investigación o satisfacción por ver que su testimonio puede tener incidencia política.

Teniendo en cuenta todo lo planteado hasta ahora en lo relativo a la investigación feminista, sus métodos y las cuestiones éticas que se plantean, el equipo de investigación se veía ante una serie de cuestionamientos a que responder, de cuya respuesta dependía la toma de decisión acerca de las técnicas utilizadas pero también sobre el proceso en cual serían implementadas dichas técnicas. La primera pregunta y más importante ya la hemos mencionado anteriormente 
¿cómo incluir en el proceso de investigación el impacto negativo que la propia indagación en la experiencia de detención pueda tener para las protagonistas? Esta cuestión incluía una segunda pregunta ¿como atenuar las relaciones de poder que se producen en toda investigación entre las investigadoras y las investigadas? A menudo las investigaciones solo incluyen las voces de las personas participantes en el momento del trabajo de campo, desapareciendo de escena sin que las investigadas tengan noticia de lo que ha ocurrido con la investigación y sin oportunidad de evaluar el impacto que dicha pesquisa ha podido dejar en las vidas de los sujetos. Y en tercer lugar, ¿qué técnica es la más adecuada para investigar a mujeres en detención policial teniendo en cuenta los límites concretos de la investigación pero aspirando a potenciar lo máximo posible la consideración de las participantes como sujetos más que como objetos en la investigación?

Una entrevista informal en la fase de pretest con una mujer que había pasado por la experiencia de detención policial dio pistas para considerar los posibles impactos que puede tener en las participantes revivir el pasaje de detención. El encuentro también nos animó a flexibilizar el planteamiento metodológico. Por otra parte, solicitamos el asesoramiento de (Emakunde-Instituto Vasco de la Mujer), investigadora conocedora de las producciones narrativas para que nos asesorase sobre la posibilidad de implementación de esta técnica en nuestra investigación, así como sobre las cuestiones que teníamos sobre la mesa.

Finalmente, y tras descartar la técnica de las producciones narrativas, nos inclinamos por la entrevista en profundidad semiestructurada, acompañada grupo de cierre al finalizar la fase de entrevista. El grupo de cierre tenía como objetivo principal dialogar sobre la experiencia de relatar la detención (qué impacto personal, subjetivo y social generó, qué emociones emergieron, etc.), de manera que pudiésemos valorar el impacto de la investigación y generar un espacio donde cerrar colectivamente el proceso de investigación entre las participantes. No obstante, los objetivos planteados para grupo de cierre se vieron desbordados, llegando a cubrir interesantes dimensiones no contempladas inicialmente por el equipo investigador, ya que nos permitió comprobar que una propuesta grupal permite colectivizar y validar las experiencias individuales de cada una de las participantes y favorecer un espacio de confianza y apoyo mutuo. A continuación, se dan más detalles sobre el contexto de la investigación, las decisiones tomadas respecto a la metodología del estudio y el grupo de cierre.

\section{MÉTODOS PARA LA INVESTIGACIÓN FEMINISTA DE LA EXPERIENCIA DE DETENCIÓN POLICIAL A MUJERES}

La investigación se realiza en el marco de las becas que anualmente ofrece Emakunde-Instituto Vasco de la Mujer con el objetivo de investigar diferentes dimensiones relacionadas con las mujeres y el feminismo. En el caso de la beca tipo 2 que nos fue concedida, estaba orientada al "análisis de las situaciones y de las desigualdades en los colectivos de mujeres en riesgo de discriminación múltiple, especialmente en el acceso a los recursos sociales, económicos y en la segu- 
ridad", tal y como consta en sus bases (Emakunde, 2016). El apoyo económico es fundamental para llevar a cabo un trabajo de estas características al tiempo marca las coordenadas de la investigación, estableciendo un límite temporal de 10 meses para su realización (de julio de 2016 a abril de 2017, concretamente), que culmina en la escritura de un informe de investigación, acompañado de recomendaciones de actuación para la mejora de la atención de las mujeres por parte de los cuerpos policiales (Zuloaga, De Miguel y Ortubay, 2017).

El equipo de investigación se conforma íntegramente por mujeres que trabajamos en la universidad, en diferentes disciplinas de la sociología y las ciencias jurídicas, posicionadas desde los conocimientos situados y parciales de Donna Haraway (1991), reconociendo que investigamos y conocemos a través de contextos culturales y de trabajo concretos (académicos, comprometidos con la transformación de la realidad), marcados por el bagaje que proporcionan trayectorias profesionales y personales particulares y por los intereses explícitos de investigación. El contexto académico genera las posibilidades para llevar adelante la empresa, al tiempo que limita, dadas las características de las instituciones universitarias actuales, cada vez más precarizadas, competitivas, burocratizadas y exigentes, donde casi todas las componentes del equipo se encuentran en posiciones de fragilidad, debido a que no cuentan con puestos estables. Además, las investigadoras afrontan el reto interdisciplinario de conformar un equipo con perfiles provenientes de la sociología y el derecho, imprescindibles para analizar la realidad de la detención policial, así como de la psicología, para valorar el impacto subjetivo, tanto de la experiencia del paso por comisaria como detenidas, como del impacto de la propia investigación. Un marco interdisciplinar que nos pone ante el reto de cruzar fronteras disciplinarias para hacernos entender y generar un conocimiento híbrido.

El compromiso feminista por la lucha contra las desigualdades sociales de género y la visibilización de las mujeres de los colectivos más vulnerables es parte relevante de nuestro conocimiento situado, que en este contexto se concreta en tratar desvelar los procesos de construcción de la mujer criminal, así como analizar críticamente el papel de las diferentes agencias penales en los procesos de criminalización de las mujeres delincuentes. Precisamente, las mujeres que han sido detenidas por la policía están habitualmente afectadas por diversos ejes de desigualdad social (género, la clase social, la etnia o raza, procedencia geográfica, etc.). Ello es debido a los procesos de selección penal (Manzanos, 1991) que operan a la hora de identificar determinados perfiles como delincuentes más a menudo que otras personas pertenecientes a grupos privilegiados.El ya clásico trabajo de (Cohen, 1967/2007) describe el proceso de construcción social de lo que denomina "demonios sociales", que son determinados acontecimientos, personas o grupos sociales son definidos como amenazas a los valores de la sociedad generando una reacción de "pánico moral" entre la población general. Estas personas y grupos, y los acontecimientos relacionados con ellos, forman parte de aquellos estigmatizados socialmente y con menos poder. Efectivamente, aunque las personas de elevada condición socioeconómica también cometen delitos, y muchos como ya observó Sutherland (1949/1999), en la cárcel no cumplen 
condena la totalidad de los delincuentes y quienes terminan en prisión comparten ciertas características que les distinguen de otro tipo de delincuentes con especial facilidad para eludirla: estatus socioeconómico, respetabilidad social, grupo étnico, estabilidad emocional, lugar de nacimiento, etc. La criminología crítica ha descrito estos mecanismos, poniendo de manifiesto la distancia entre la criminalidad y la persecución penal (González Sánchez, 2015; Larrauri 1992), de manera que medir e interpretar la criminalidad de una sociedad sobre la base de sus estadísticas carcelarias es un error que distorsiona tanto como hacerlo sobre el número de detenciones realizadas por la policía. Los resultados cuantitativos, en ambos casos, no constituyen el reflejo de la delincuencia real que experimenta una sociedad, sino que son la consecuencia de la política criminal que se practica, es decir, de la reacción política acordada para enfrentarla (Zuloaga 2014). En palabras de Barañí, "el sistema punitivo, lejos de funcionar como reacción automática a la criminalidad, como muy a menudo se cree, es el producto de un gran número de decisiones tomadas por los y las profesionales de las instancias que intervienen en él" (2001:74).

Los estudios sobre mujeres encarceladas, con todas las diferencias que puede haber respecto a las estadísticas policiales, ya que hablamos de dos momentos diferentes del sistema penal, vienen a confirmar esta persecución de las capas más vulnerables y estigmatizadas de nuestra sociedad (Almeda 2005; Barañi 2001; Cruells y Torrens 2004; De Miguel Calvo 2014; Imaz y Martin-Palomo 2007; Mapelli Cafarena et al. 2012; Ribas, Almeda, y Bodelón 2005), mostrando un gran número de mujeres pobres entre rejas, así como la sobrerrepresentación de extranjeras (una de cada tres había nacido fuera de nuestras fronteras), la alta presencia de (ex)toxicómanas (casi la mitad) o el elevado porcentaje de las que pertenecían a una etnia no mayoritaria, concretamente gitanas.

Estamos en un escenario, por tanto, de mujeres investigando a mujeres, con todas las posibilidades que ello conlleva de poder generar entornos de confianza (Oakley 1981), pero que al mismo tiempo conlleva no pocas ambigüedades. Una de estas ambigüedades es la que deriva del riesgo de explotar esa relación básicamente jerárquica entre participantes e investigadoras para intereses no siempre relacionados con los intereses de las mujeres protagonistas (Finch 1984), ya que como señalábamos anteriormente, aunque se intenten suavizar las aristas de las relaciones de poder entre investigadoras y participantes, estos desequilibrios no desaparecen. El reto está en diseñar metodologías "amables" que rompan en la medida de lo posible las dicotomías sujeto/objeto de investigación como dirían Gandarias y García Fernández (2015). De ahí que se considerase la posibilidad de usar el método de las producciones narrativas en la presente investigación.

Las producciones narrativas (en adelante PN), tal y como describe Itziar Gandarias (2014b), forman parte de los métodos narrativos en el contexto del "giro discursivo" de que dan cuenta Denzin y Lincoln (2012). Esta herramienta se viene desarrollando desde finales de los 90 por el grupo de investigación FICFractalidades en Investigación Crítica del Departamento de Psicología Social de la UAB (Gandarias y García Fernández 2015:97). Se trata de un planteamiento de investigación dialógico y no lineal, de carácter feminista e interseccional en 
la medida que valora la posición epistémica de sujetos tradicionalmente invisibilizados, que busca potenciar la agencia de las participantes y que tiene como objetivo transformar las relaciones de poder generadas por diferentes ejes de opresión (Gandarias Goikoetxea, 2014b:130).

El planteamiento se concreta en la "producción conjunta de un texto híbrido construido conjuntamente a partir de a) sesiones donde la investigadora y participantes hablan y discuten distintos aspectos del fenómeno que se quiere estudiar, b) la textualización, que funcionaría como una revisión y reflexión sobre la sesión, en que en el que la conversación se traduce a un texto organizado y comunicable que refleja las posiciones y argumentos desarrolladas a lo largo de la sesión, y c) el reconocimiento de la agencia de las participantes para modificar, corregir, expandir la textualización realizada hasta que validen la narrativa creada» (Gandarias Goikoetxea 2014b:131).

Las PN no son consideradas material empírico a analizar, sino puntos teóricos de partida, permitiendo mayor control de las protagonistas de lo que quieren que sea comunicado acerca de una determinada cuestión, atenuando la contradicción que supone para las investigadoras dedicarse a "la discutible labor de ir descifrando las palabras ajenas desde una institución académica" como expresa Spivak (2002:213). De hecho, los textos permiten abrir espacios de producción de conocimiento más allá de la academia, debido a su carácter autónomo respecto a la investigación (Gandarias Goikoetxea, 2014b). Las investigadoras juegan el papel de facilitadoras del diálogo y líderes en proceso de elaboración del texto, desde un rol particular que muestra la multiplicidad de saberes que se ponen en juego. Esta herramienta metodológica, por tanto, permite aplicar principios fundamentales de la metodología feminista, articulando una propuesta que permite otorgar mayor capacidad de control a las participantes acerca del texto final del proceso de investigación y mayor voz en el proceso de investigación, dado que la mediatización de sus voces es menor.

Pero las PN presentan algunas debilidades o "limirretos" a la hora de implementarse con mujeres en situación de vulnerabilidad (Gandarias y García Fernández 2015). Exigen un grado de compromiso y dedicación que muchas mujeres no están en condiciones de ofrecer, bien porque se encuentran en situaciones de precariedad laboral o por precariedades subjetivas y personales derivadas de desventajas sociales. Además, requieren de un dominio lingüístico y de escritura que hace difícil la participación a determinados perfiles de mujeres, como puede ser el caso de aquellas que no conocen el idioma autóctono o quienes tienen bajo nivel de alfabetización.

Gandarias describe las dificultades de implementación de las PN en el estudio de la experiencia de migración de mujeres subsaharianas de la siguiente manera:

"La mayor dificultad que encontramos fue establecer con ellas más de una sesión. Una de las participantes, durante el proceso de construcción de la narrativa, consiguió un trabajo fuera del País Vasco y fue imposible poder llevar a cabo nuevas sesiones. En este caso, la validación de la narrativa se hizo por teléfono y por correo electrónico. Otra dificultad ha sido el idioma. 
Escribir las narrativas en castellano ha condicionado el diálogo de negociación conjunta de las mismas, ya que al no dominar el idioma se sentían limitadas para realizar cambios al texto"(Gandarias Goikoetxea, 2019:8).

Efectivamente, extender el uso de la técnica de PN de mujeres activistas (Gandarias Goikoetxea, 2014; García Fernández \& Montenegro Martínez, 2014) al caso de mujeres especialmente vulneradas puede no ser lo más adecuado. Teniendo en cuenta que en el caso de las mujeres en detención policial era muy habitual encontrarnos con personas extranjeras que pudiesen algunas de ellas tener dificultades de comunicación oral o escrita (teniendo en cuenta que las producciones narrativas consisten en la elaboración de un texto escrito) o que no cuenten con las condiciones materiales necesarias como para dedicar mucho tiempo y esfuerzo a la construcción de las producciones narrativas, el equipo investigador optó por recurrir a la técnica de la entrevista. A ello se le sumaban las limitaciones materiales y temporales con que las investigadoras contaban debido a los parámetros institucionales que se situaban y que hemos descrito anteriormente.

La entrevista semiestructurada no permite que las entrevistadas puedan controlar lo que ellas han dicho, más allá del momento de la realización de la entrevista, ni que la autoría del texto final sea de las protagonistas. Cierto que, con cada vez mayor frecuencia, se está dando la posibilidad de que quien participa en una entrevista acceda a la transcripción, con el fin de que pueda matizar o rectificar ciertas ideas que se expresaron. Sin embargo, el texto final ya no es coproducido entre investigadores y participantes como el caso de las PN sino que en la entrevista los roles de ambas partes están más diferenciados y las asimetrías aparecen más marcadas.

La herramienta no es el único aspecto a dilucidar, ya que la forma en que se aplique marcará un estilo y un hacer determinado, que se encuentra más o menos en consonancia con el marco epistemológico y metodológico. En nuestro caso, la técnica de la entrevista se aplicó de una manera poco directiva y estructurada (Taylor y Bogdan 1996), ya que de esta manera posibilita abordar los temas planteados, dando la opción a la espontaneidad de las mujeres entrevistadas, abordando también los diferentes temas que surgen alrededor del tema principal e incluso parando la entrevista si las participantes lo ven necesario

El grupo de cierre, por su parte, también contiene unas virtualidades que lo hacen coherente con las líneas metodológicas feministas. En la búsqueda de sopesar las consecuencias que relatar la experiencia de paso por comisaría había podido tener para las protagonistas, dando un cierre a su participación en la investigación, las investigadoras asumen la responsabilidad de las consecuencias de la investigación considerando el posible daño que ésta cause a las participantes, teniéndoles en cuenta como sujetos. El grupo de cierre, además, permitió colectivizar la experiencia individual de la estancia en calabozo y generar significados a partir de la interacción. 


\section{EL GRUPO DE CIERRE: CERRAR EL PROCESO, CONTRASTAR RESULTADOS Y MUCHO MÁS.}

En su investigación sobre tortura y malos tratos en el País Vasco, Etxeberria, Martin Beristain y Pego (2017) desarrollaron grupos de cierre (en adelante GC) para valorar el impacto que tuvo la participación en quienes contaron sus experiencias como víctimas. Aunque fue llevado adelante por el equipo de psicólogas de la investigación, aclaraban que "Se trata de sesiones grupales, no eran sesiones de terapia. Eran grupos de acompañamiento para valorar cómo se encontraban y ayudarles a colocar todo aquello que se había movido en la entrevista (Etxeberria, Martin Beristain y Pego 2017:196). En base a esa experiencia, el equipo investigador optó por aplicar el GC a la investigación de la experiencia de detención policial a mujeres. Convocamos a las 9 que participaron a través de las entrevistas en nuestra investigación con el mismo objetivo: acompañar la "digestión" de la experiencia de narrar y narrar-se, considerar el daño potencial de la investigación en las participantes. Además, teniendo en cuenta que el informe se encontraba en fase de finalización, consideramos que era una oportunidad inmejorable para compartir y contrastar los resultados más relevantes de la investigación. Finalmente, tres mujeres que habían sido entrevistadas acudieron al local cedido por una entidad social de una de las capitales vascas, el día y hora acordados. El resto de participantes había mostrado gran interés en participar, pero diversas ocupaciones o cuestiones derivadas de su vulnerabilidad social impidieron que acudieran a la cita, ya fuera trabajo, imprevistos caseros de última hora o diversos malestares personales derivados de situaciones de exclusión social . Ciertamente, uno de los límites de las técnicas grupales reside en la dificultad de reunir a varias personas en el mismo lugar el día y hora señalado. La sesión fue coordinada por una investigadora del proyecto y una colaboradora. Las participantes en el grupo de discusión fueron Ada, Tania y Rebeca ${ }^{2}$.

Ada tenía 60 años de edad y había estado detenida por la policía en numerosas ocasiones por delitos contra la salud pública, sobre todo en comisarías de la Ertzaintza. Tania, extranjera latinoamericana de 40 años y residente en una de las capitales de provincia vascas, pasó por comisaría en dos ocasiones, una por la policía municipal y otra por la Ertzaintza, acusada de sendos delitos de lesiones. Y Rebeca es una mujer de 42 años que había sufrido numerosas detenciones relacionadas con su adicción a las drogas. Las tres han estado encarceladas y se conocieron en la misma prisión.

En cuanto a lo que supuso narrar la experiencia de la estancia en comisaría como detenida, ninguna de las participantes destacó que hubiese tenido un impacto negativo, más bien al contrario, hablaron de que sirvió para liberar de alguna manera esa experiencia. Así, Tania afirmó que le pareció bien hablar de ello, "soltar un poquito mi experiencia. (...) No fue traumático ni nada de esas cosas". Rebeca, por su parte, cuenta que es beneficioso para ella tener la oportu-

\footnotetext{
${ }^{2}$ Los nombres de las participantes son ficticios.
} 
nidad de hablar de ello: "Son experiencias que las necesito sacar, pero con esta (experiencia de detención), por ejemplo, no se ha dado la oportunidad de sacarla muchas veces, en petit comiteé, pues con cierta gente, pues si he podido explicar, hasta donde yo recuerdo, porque he intentado dejarlo en el pasado".

La percepción que se tiene del GC es de un contexto seguro, tal y como transmitieron en el tono de sus intervenciones y en la actitud que mostraron, como por lo que se puede deducir de las palabras de Rebeca: "aquí me siento, en principio, protegida y muy a gusto. Muy tranquila.

A medida que aparecían diferentes cuestiones referidas a la estancia en calabozo, fue posible que entre ellas mismas contrastaran sus experiencias con las demás:

Ada: "Yo a lo mejor, pues, yo le decía: mira que me ha bajado el periodo. Y me traía una compresa y una braguita y me decía: ¿Quieres ducharte?"

Rebeca: ¿Ducharte en comisaría?

Ada: "Sí, si te daban un chandal y te daban playeras. Aquí en la Ertzaina. En la Ertzaina, en la (policía) municipal no. La Ertzaina sí. Tú le dices a la ertzaina: Mira por favor me ha bajado la regla. Y te traía una compresa y una braga”.

Otra dimensión del contraste es la que se refiere a comprobar si las propuestas que cada una de ellas hicieron de manera individual tienen eco en el grupo. Esto ocurrió con la propuesta de Tania de contar con asistencia psicológica durante la detención, algo que fue bien visto por las otras dos compañeras:

Tania: "uno de los temas que yo te había dicho cuando hicimos la entrevista, un poco el psicólogo (...) ya estás hundida porque estás allí encerrada te acaban de hundir, te acaban de hundir, entonces que el psicólogo esté, aunque sea para que te mienta, aunque sea para que te mienta en ese momento, para que las ideas de suicidio se te desaparezcan.

Rebeca: Es súper importante, me parece a mí.

Tania: ¿A que sí? (...) Mira ¿ves? Ella me apoya ahí con la idea.

El equipo investigador valoró esta propuesta tan aplaudida por las asistentes al grupo, pero finalmente declinó la posibilidad de introducirlo en la batería de propuestas del informe, en base a la idea de que el proceso de detención debe ser lo más breve posible, de manera que la persona detenida salga de comisaría a la calle o pase a disposición judicial. Consideramos, no sin poco debate entre las participantes del equipo investigador, que el calabozo no es el lugar apropiado para que una persona profesional de la psicología haga una intervención. Esto muestra las diferencias que puede haber entre la perspectiva de las participantes y de las investigadoras, y cómo la última palabra en este caso estuvo en manos de las responsables del estudio, expresando así las desigualdades de poder que hemos señalado anteriormente.

Llegó un momento en que las investigadoras estaban imbuidas en el tema, hasta el punto de plantearse la tesitura de estar ellas mismas en un calabozo. Hablando sobre la práctica de retirar las gafas, entre otros objetos que se retiran aduciendo que pueden ser usados para autolesionarse, esta investigadora dice: 
"sé de lo que habláis porque soy miope también yo, tengo lentillas. Que también alguna vez he pensado ¿Si me detienen? Toco madera... (golpea la mesa). Pero yo no puedo tener las lentillas toda la noche, entonces bueno, pues no sé qué pasa en esos (casos) (...) yo no había pensado en las lentillas hasta que salió el tema de las gafas". Paradójicamente, el momento más hilarante de la reunión fue aquel en que todas nos encontramos imaginando las situaciones más comprometidas que se pueden producir estando detenida:

Esti [Investigadora]: (A Tania) Me acuerdo que comentaste "cualquier cosa menos un agujero de esos para hacer las necesidades.

Tania: “...Ay, sí. Es horrible lo del agujero ¿no te parece? Es horrible el agujero".

Esti [Investigadora]: (Ríe) (A Rebeca) ¿De qué te ríes ?

Rebeca: Porque lo estoy viendo, ja ja.

Ada: Fíjate que en ese momento hubieses tenido diarrea.

Itziar [Investigadora]: Eso estaba pensando yo también, ja ja.

Ada: Fíjate tú, qué horrible, es que es horrible.

Tania: Quítate, ese olor a berrín, a meao ¡Qué asco!

Itziar [Investigadora]: .... a pulso...

Ana: Tienes que mear medio de pie, imagínate si tienes diarrea.

Itziar [Investigadora]: A pulso, ja ja.

Efectivamente, el humor estuvo presente en diversos momentos de la sesión, lo cual da cuenta de lo relajado del ambiente y de lo seguras que las participantes se sintieron.

Tania: La atención de la (policía) municipal muy mala ¿eh? ¡Yo a ese hotel no vuelvo! (ríe)

También hubo apertura a compartir otras emociones, que fueron acompañadas por las demás. Las tres invitadas hablaron de cuestiones de sus vidas con una fuerte carga emocional, relacionadas de manera indirecta con el objeto de la reunión. En el caso de Ada, transmitió su sentimiento de culpa por haberse dedicado a traficar con drogas durante buena parte de su vida:

Ada: (...) me arrepiento porque cuántos habrán muerto por venderles yo la "mierda" pero bueno. Es lo único porque...como yo no sé si han muerto por mí o porque...pues sí, yo he hecho daño (...) Yo veo que estoy matando a gente, pero...bueno, tampoco les ponía un puñal para que me la compraran a mí, pero en un momento sí te sientes como culpable.

Tania: Yo no lo vería por ese punto porque al final todos somos adultos. Si vas y lo vendes a un niño que no sabe lo que hace, estaría mal. Pero a un adulto, que sabe lo que hace, que si no te lo coge a ti va a conseguirlo en otro sitio, pues...

Ada: En cierta parte también te sientes como...como culpable. Es lo que tú dices, que si no se lo vendes tú, o se lo vende la otra o se lo vende la otra. A ver si me entiendes porque esto es así, en el mundo de la droga es así. Si no soy yo es otro, o sea, que da lo mismo. Pero en cierto modo dices iy si pudiera ser un familiar mío? ¿Y si pudiera ser un...? Claro, en estos momentos piensas, pero en esos momentos no piensas. Dices, "hala, toma, y punto". Yo 
digo, es de lo único que me arrepiento.

Finalmente, las cuestiones no solo fueron lanzadas desde las investigadoras hacia las protagonistas, sino que ellas también tenían preguntas acerca de los términos de la investigación: ¿cómo se ha hecho la contactación, siendo un tema tan delicado?, ¿se ha accedido a expedientes policiales? ¿qué pasa con las entrevistas, esto a dónde va? ¿es la primera vez que se hace? A la última pregunta se respondió que esta es una investigación pionera en el País Vasco y en el Estado español, a lo que Tania replicó con cierto orgullo: “Ah, mira, somos pioneras», expresando un alto nivel de identificación con la investigación.

Así, narrar la experiencia de detención policial no había supuesto un daño en las mujeres que formaron parte del GC, más bien al contrario, son experiencias que se necesitan compartir. Aunque bien es cierto que es necesario matizar esta aseveración debido al reducido número de participantes, que impide hacer generalizaciones y requiere de que futuras investigaciones desarrollen esta técnica. El GC sirvió también como espacio para que las investigadoras hicieran una devolución de los resultados, en un momento en que el informe con las conclusiones de la investigación estaba cercano a presentarse públicamente. Las protagonistas mostraron gran interés por conocer tanto el contenido del mismo como los pasos que se habían dado y los que se iban a dar en adelante. A este respecto, la devolución de resultados supuso que el intercambio investigadoras/investigadas ofreciera la posibilidad de generar una cierta reciprocidad, a pesar de los diferentes puntos de partida e intereses de ambas partes. Pero el planteamiento del grupo de cierre desbordó con creces los objetivos planteados, al generarse un espacio "amable" donde compartir vivencias, emociones y temores relacionados con la experiencia de criminalización y el paso por el sistema penal. Se comprobó el gran potencial de las herramientas dialógicas para el conocimiento, la validación y la colectivización de experiencias que tienden a ser desconocidas por su alto grado de estigmatización.

\section{CONCLUSIONES}

La investigación de la experiencia de detención policial a mujeres en la Comunidad Autónoma Vasca, se sostiene sobre unas bases feministas, en lo referente al marco teórico de la criminología feminista interseccional, sino también en lo referente a la epistemología y metodología. A este respecto, el objetivo se orienta a desvelar las desigualdades de género en lo referente a la práctica policial de detención, otorgando relevancia a la experiencia de las mujeres detenidas en primera persona. Al tratarse del estudio de los primeros eslabones de la selección penal, nos encontramos no sólo con desigualdades de género, sino también con la intersección de diversos ejes de estratificación social (clase social, etnia, procedencia geográfica, etc.).

La metodología feminista se caracteriza por primar la experiencia de las mujeres, eterna olvidada de la ciencia androcéntrica, reconociendo que todo 
quehacer científico es un acto político, que requiere asumir responsabilidad. De ahí que la dimensión ética cobre especial relevancia, incluyendo la atención a unas relaciones de poder, que se producen a diferentes niveles. Especialmente destacable es la interacción entre investigadoras e investigadas, una relación de partida jerárquica pero que es posible amortiguar teniendo en cuenta las diversas decisiones tomadas en el proceso y el reconocimiento de la agencia de las mujeres participantes.

Pero la elección de las herramientas y de la forma de aplicarlas no se produce en situaciones ideales, sino en contextos marcados por las limitaciones institucionales de la academia, por las condiciones establecidas por las agencias que proporcionan el apoyo económico, o por los propios condicionantes de las participantes. De ahí que descartáramos un método "amable", que en principio ofrece numerosas posibilidades para atenuar la distancia objeto/sujeto, en favor de la entrevista semiestructurada. Aun así, en la búsqueda de atenuar el daño que la narración de la experiencia de detención policial, siempre estigmatizante, pudiese generar, nos inclinamos por un grupo de cierre. Esta reunión grupal desbordó los objetivos y generó unas dinámicas en las que las investigadoras se vieron imbuidas. Además de valorar el poso que la entrevista había dejado y de comentar resultados de la investigación, las protagonistas tuvieron la oportunidad de devolver preguntas a las investigadoras en lo que respecta a los términos de la investigación, y hubo espacio para la validación mutua, para compartir experiencias propias de alta densidad emocional, incluso de reír juntas, en un clima considerado seguro. Resultando, así, que es posible desarrollar métodos que, sin eliminar por completo las jerarquías, generen contextos de conocimiento más horizontales, donde las investigadoras y las investigadas tienen roles y responsabilidades diferentes, pero pueden situarse en una cercanía y sentirse identificadas. Todo ello muestra que es en la interacción donde se genera el conocimiento y que las emociones son parte sustancial de las experiencias que merece la pena comunicar.

\section{BIBLIOGRAFÍA}

ABAD, B. (2016). "Investigación social cualitativa y dilemas éticos: De la ética vacía a la ética situada", Empiria. Revista de Metodología de Ciencias Sociales, 34, pp. 101-120. https://doi.org/DOI/empiria.34.2016.16524

ACKERLY, B., \& TRUE, J. (2010). «A feminist research ethic explained» en Doing feminist research in political and social science, London, Palgrave, pp. 21-39.

ALMEDA, E. (2005). «Women's imprisonment in Spain», Punishment and Society, (7), pp.183-199. https://doi.org/10.1177/1462474505050442

ALMEDA, E. (2017). «Criminologías feministas, investigación y cárceles de mujeres en España». Papers. Revista de Sociologia, 102(2), pp. 151-181.

ASAMBLEA GENERAL DE LA ONU. (2018). "Informe del Grupo de Trabajo de Expertos sobre los Afrodescendientes acerca de su misión a España", disponible en página web [consulta: 26-May-2019] http://www.rightsinternationalspain.org/ uploads/publicacion/eca132299837fd31773b149f2e9d600c5142af6c.pdf 
BALLESTEROS-PENA, A. (2019). Facing the Diversity of Carceral Environments in Prison Research: Lessons From my Fieldwork with Women in Three Prisons in Spain. Cadernos Pagu, 55, 1-25. https://doi.org/10.1590/18094449201900550004

BARAÑI, E. (2001). "Mujeres gitanas y sistema penal" Madrid, Metyel.

BARBERET, R. (2014). "Women and offending" en Women, Crime and Criminology, London and New York, Routledge, pp.137-158

BIGLIA, B. (2015). "Avances, dilemas y retos de las epistemologías feministas en la investigación social" en Otras formas de (re)conocer, Bilbao, Vitoria-Gasteiz y Donostia, Hegoa y Simref, pp. 21-44.

BVODDHH. (2013). Cuando la vulneración de derechos se normaliza. Controles de identidad racistas en Madrid. $2^{\circ}$ informe de las Brigadas Vecinales de Observación de DDHH (2011-2012). http://library1.nida.ac.th/termpaper6/sd/2554/19755.pdf

COMITÉ PARA LA PREVENCIÓN DE LA TORTURA (2017). Informe para el Gobierno Español sobre la visita llevada a cabo en España por el Comité Europeo para la Prevención de la Tortura y de las penas y tratos inhumanos o degradantes (CPT), disponible en página web [consulta: 26-May-2019] http://www.infocoponline.es/ pdf/cpt1.pdf

CRUELLS, M., \& TORRENS, M. (2004). "Mujeres, integración y prisión. Un análisis de los procesos de integración sociolaboral de las mujeres presas en Europa”, Barcelona, Aurea.

DE MIGUEL CALVO, E. (2014). "Encarcelamiento de mujeres. El castigo penitenciario de la exclusión social y la desigualdad de género" Zerbitzuan, 56 (Septiembre), pp. 75-86. https://doi.org/10.5569/1134-7147.56.05

DE MIGUEL CALVO, E. (2016). "Relaciones amorosas de las mujeres encarceladas", Bilbao, UPV/EHU Servicio Editorial.

DENZIN, N. K., \& LINCOLN, Y. S. (2012). "Manual de investigación cualitativa”, Madrid, Gedisa.

DICKSON-SWIFT, V., JAMES, E. L., KIPPEN, S., \& LIAMPUTTONG, P. (2008). Risk to researchers in qualitative research on sensitive topics: issues and strategies. Qualitative Health Research, 18(1), 133-144. https://doi. org/10.1177/1049732307309007

EMAKUNDE. "Becas de trabajos de investigación en materia de igualdad de mujeres y hombres para el año 2016", disponible en página web [consulta: 26-May-2019] https://www.euskadi.eus/web01-tramite/es/contenidos/ayuda_subvencion/becas_2016/es_def/index.shtml

ENGLAND, K. V. L. (1994). «Getting personal: reflexivity, positionality and feminist research», The Professional Geographer, 46(1), pp. 80-89.

ETXEBERRIA, F., BERISTAIN, C. M., \& PEGO, L. (2016). «Proyecto de investigación de la tortura en el País Vasco (1960-2013)», disponible en página web [consulta: 26-May-2019] http://www.eitb.eus/multimedia/documentos/2016/06/27/1987310/Memoria Proyecto tortura 2016.pdf

FINCH, J. (1984). "It's great to have someone to talk to" en Social Researching: Politics, Problems, London, Routledge and Kegan Paul, pp.71-87

GANDARIAS GOIKOETXEA, I. (2014a). "Habitar las incomodidades en investigaciones feministas y activistas desde una práctica reflexiva", Athenea Digital. Revista de Pensamiento e Investigación Social, 14(4), pp. 289-304. https://doi.org/10.5565/rev/ athenea.1489

GANDARIAS GOIKOETXEA, I. (2014b). "Tensiones y distensiones en torno a las relaciones de poder en investigaciones feministas con Producciones Narrativas”, Qua- 
derns de Psicologia, 16(1), pp. 127-140. https://doi.org/10.5565/rev/qpsicologia. 1210

GANDARIAS GOIKOETXEA, I. (2019). Resistir desde la vulnerabilidad: Narrativas de mujeres subsaharianas sobre su tránsito hacia Europa. Papeles Del CEIC, 2019(1), 1-18. https://doi.org/10.1387/pceic.19532

GARCÍA FERNÁNDEZ, N., \& MONTENEGRO MARTÍNEZ, M. (2014). Re/Pensar las producciones narrativas como propuesta metodológica feminista: experiencias de investigación en torno al amor romántico. Athenea Digital. Revista de Pensamiento e Investigación Social, 14(4), 63. https://doi.org/10.5565/rev/athenea.1361

GANDARIAS, I., \& GARCÍA FERNÁNDEZ, N. (2015). "Producciones narrativas: una propuesta metodológica para la investigación feminista" en Otras formas de (re)conocer, Bilbao, Vitoria-Gasteiz y Donostia, Hegoa y Simref, pp.- 97-110

GOFFMAN, E. (2001). Internados. Ensayos sobre la situación social de los enfermos mentales $\left(1^{\mathrm{a}}\right.$ en cast). Amorrortu.GONZÁLEZ SÁNCHEZ, I. (2015). La penalidad neoliberal: aumento de presos y reconfiguración del Estado en España (1975-2008). Tesis Doctoral. Universidad Complutense de Madrid.GRAU, E. (2015). "Saber que alguien lo escucha. El método de la narrativa en la investigación. La verdad de las mujeres. Víctimas de conflicto armado en Colombia" en Otras formas de (re)conocer, Bilbao, Vitoria-Gasteiz y Donostia, Hegoa y Simref, pp. 147-165HAMMERSEY, M. (1992). "On feminist methodology» Sociology, 26(2), pp. 187-206.HARAWAY, D. (1991). "Conocimientos Situados: la cuestión científica en el feminismo y el privilegio de la perspectiva parcial" en Ciencia, cyborgs y mujeres, Madrid, Cátedra, pp. 313-346HARDING, S. (1987). "Is there a feminist method?» In Feminism and Methodology, Bloomington, Indiana University Press, pp. 1-14

HEIMER, K., \& KRUTTSCHNITT, C. (2005). «Gender and Crime. Patterns in Victimization and Offending» New York, New York University Press.

IMAZ, E., \& MARTIN-PALOMO, T. (2007). "Las otras otras: extranjeras y gitanas en las cárceles españolas" en Estado de Wonderbra: Entretejiendo narraciones feministas sobre las violencias de género, Madrid, Virus, pp. 217-227

JEWKES, Y. (2011). «Autoethnography and emotion as intellectual resources: doing prison research differently», Qualitative Inquiry, 1(18), pp. 63-75.

JULIANO, D. (2009). "Delito y pecado. La transgresión en femenino", Política y Sociedad, 46(1), pp. 79-95

LARRAURI, E. (1992). "La herencia de la criminología crítica", Madrid, Siglo XXI.

LIPSON, J. G. (2003). "Asuntos éticos en la etnografía" In J. Bottorf (Ed.), Asuntos críticos en los métodos de investigación cualitativa, Medellín, Universidad de Antioquía, pp. 389-415.

MALLOCH, M. S. (2000). "Women, drugs and custody" Winchester: Waterside Press.

MANZANOS, C. (1991). "Cárcel y marginación social. Contribución crítica e investigación aplicada a la sociedad vasca" Donostia, Gakoa.

MAPELLI CAFARENA, B., HERRERA MORENO, M., GUTIÉRREZ ROMERO, F., AGUDO CORREA, T., \& SORDI STOCK, B. (2012). "Mujeres en las cárceles de Andalucía" Madrid, Dykinson.

MEO, A. I. (2010). "Consentimiento informado, anonimato y confidencialidad en investigación social. La experiencia internacional y el caso de la sociología en Argentina" Aposta. Revista de Ciencias Sociales, 44, pp. 1-30.

MIRANDA, M. J., \& SÁNCHEZ, I. G. (2018). "Academia, cárcel y relaciones de poder. Entrevista con $\mathrm{M}^{\mathrm{a}}$ Jesús Miranda" Encrucijadas - Revista Crítica de Ciencias Sociales, 16, pp. 1-16. 
OAKLEY, A. (1981) «Interviewing women: a contradiction in terms» en Doing Feminist Research, London, Routledge, pp. 30-61.

ORGAZ ALONSO, C. (2018). Emergencia del dispositivo deportador en Europa y su generalización en el caso español: representaciones y prácticas en torno a los Centros de Internamiento para Extranjeros (CIE). Universidad Complutense de Madrid.PEGO OTERO, L. (2017). "Justicia Transicional: Los derechos de las víctimas por la aplicación indebida de la detención incomunicada". University of the Basque Country, disponible en página web [consulta: 26-May-2019] https://addi. ehu.es/bitstream/handle/10810/26732/TESIS_PEGO_OTERO_LAURA.pdf?sequen ce=1\&isAllowed=yRAMAZNOGLU, C., \& HOLLĀND, J. (2002). «Feminist Methodology. Challenges and choices» London, Sage.RIBAS, N., ALMEDA, E., \& BODELÓN, E. (2005). "Rastreando lo invisible. Mujeres extranjeras en las cárceles", Barcelona, Anthropos.

RIS. (2019). "Balance de 2018. Los derechos humanos en España", disponible en página web [consulta: 26-May-2019] http://rightsinternationalspain.org/uploads/publicacion/dbd3ceba69fe7aa53aa3d11f188cfc673219025c.pdf

SÁDABA RODRÍGUEZ, I. (2014). "Reflexiones metodológicas sobre la investigación social en cárceles", en Una condena compartida. Un estudio de caso sobre el control penal, Madrid, Tierra de Nadie, pp. 183-216.

SAINZ DE LA MAZA QUINTANAL, E. (2015). Ultima ratio. El proceso de expulsión de inmigrantes en situación irregular en España. Tesis Doctoral. Universidad Complutense de Madrid.

SAN MARTÍN, J., ARTEAGA, M., LÓPEZ DE FORONDA, F., BERISTAIN, A., \& DE LA CUESTA, J. L. (1991). "Los calabozos. Centros de detención municipales y de la Ertzantza”. Vitoria-Gasteiz, Ararteko y Universidad del País Vasco.

SCHLOSSER, J. A. (2008). "Issues in interviewing inmates. Navegating the methodological landmines of prison research». Qualitative Inquiry, 14(8), pp. 1500-1525.

SMART, C. (1977). «Women, crime and criminology: a feminist critique» London, Routledge and Kegan Paul.

SMITH, A., \& PITTIS, M. (2005). «Researching de margins: An Introduction», en Researching the margins. Strategies for ethical and rigorous research with marginalised communities, New York, Palgrave, pp. 3-41.

SPIVAK, G. C. (2002). «¿Puede hablar la subalterna?” Asparkía. Investigació feminista, (13), pp. 207-214.

SUTHERLAND, E. H. (1999), "El delito de cuello blanco", Madrid, La Piqueta.

TAYLOR, S. J., \& BOGDAN, R. (1996). "Introducción a los métodos cualitativos de investigación", Barcelona y Buenos Aires, Paidón.

VIEDMA ROJAS, A., DEL VAL CID, C., \& CALLEJO GALLEGO, J. (2019). "Investigar en cárceles. Apuntes para futuros científicos sociales". Empiria. Revista de Metodología de Ciencias Sociales, 0(42), 165-195. https://doi.org/10.5944/empiria.42.2019.23256

WALMSLEY, R. (2015). «World Prison Female Imprisonment List-3rd Edition», disponible en página web [consulta: 26-May-2019] http://www.prisonstudies.org/sites/ default/files/resources/downloads/world female prison 4th edn v4 web.pdf

ZULOAGA, L. (2014). «El espejismo de la seguridad ciudadana», Madrid, Libros de la Catarata. 
ZULOAGA, L., DE MIGUEL, E., \& ORTUBAY, M. (2017). «Experiencia de la detención policial en las mujeres de la Comunidad Autónoma de Euskadi», disponible en página web [consulta: 26-May-2019]

http://www.emakunde.euskadi.eus/contenidos/informacion/publicaciones_bekak/es_def/ adjuntos/beca.2016.experiencia_detencion_policial_mujeres_cae.pdf 